\title{
A Novel Immunosorbent Assay for the Detection of Brominated Flanme Retardant-- Tetrabromobisphenol A
}

\author{
ZHUANG Hui-sheng, Bu Dan
}

School of Environment Science and Technology, Shanghai Jiao Tong University, No.800, Dongchuan Road, Minghang district, Shanghai 200240, P.R. China, hszhuang

\begin{abstract}
Tetrabromobisphenol A (TBBPA) is a kind of brominated flame retardants widely used in the world. A sensitive and selective competitive indirect biotin-stretavidin amplified emzyme-linked immunosorbent assay (BA-ELISA) for the detection of TBBPA was developed. The limit of detection (IC10) and the inhibition half-maximum concentration (IC50) were $0.027 \mathrm{ng} / \mathrm{mL}$ and $0.58 \mathrm{ng} / \mathrm{mL}$, respectively. Cross-reactivity values of the BA-ELISA with a set of analogues revealed a high selective. Finally, the assay was applied to the analysis of TBBPA in electronic waste samples. The results were agreed well with those of liquid chromatography method, proved that the established immunoassay were accurate and receivable. It is believed that this BA-ELISA method was suitable for rapid and sensitive screening of TBBPA in environmental monitoring.
\end{abstract}

Keywords: flame retardant, Tetrabromobisphenol A, antigen, polyclonal antibody, immunoassay, biotin-stretavidin amplified system, electronic waste.

\section{Introduction}

TBBPA had been listed in 《Convention for the protection of the marine environment of the North-East Atlantic》, as a hazardous substance. Meanwhile, researchers found that TBBPA is an endocrine-disrupting chemical ${ }^{[1]}$ because of the alike molecular structure to thyroxine. Other studies showed that it is an immunotoxic and neurotoxic compound ${ }^{[2,3]}$. And TBBPA could be dehalogenated under anaerobic and aerobic conditions to yield bisphenol A (BPA).

For determination of TBBPA and its derivatives in varieties of environmental samples, gas or liquid chromatography techniques ${ }^{[4-8]}$ were used. However, the instrumental analytical methods have their limitation, poor specificity, low sensitivity, complex pro-treatment procedures and expensive instruments, which restrict their widespread use and rapid detection of TBBPA from environmental samples. Compared to instrumental analytical methods, enzyme linked immunosorbent assay (ELISA) is well suited in detecting trace pollutant in environment because of its high specificity, sensitivity and throughput. In literature, ELISA method had been reported for measuring polybrominated diphenyl ethers (PBDEs) ${ }^{[9-13]}$, other kind of brominated flame retardants.

Hence, the aim of this study was to develop a modified indirect competitive ELISA for TBBPA, using a biotinstreptavidin amplifying system. The diverse hapten of TBBPA, immunogen and relative polyclonal antibody were prepared. And the established immunoassay procedures were optimized, which accuracy and sensitivity were compared with liquid chromatography method. We think our resulted biotinstreptavidin system amplified ELISA method (BA-ELISA) could selective and sensitivity detecting TBBPA in various environmental samples and will be helpful for environmental studies.

\section{Materials and method}

\subsection{Reagents and Apparatus}

Tetrabromobisphenol A (TBBPA) standard and organic materials for synthesizing hapten were purchased from $\mathrm{J} \& \mathrm{~K}$ Chemical. Hapten was purified by column chromatographic separation, carried out using silica gel $(40 \mu \mathrm{m}$ average particle size) from Shanghai Sanpont Co.Ltd. Bovine serum albumin (BSA), egg albumin (OVA), biotinyl- $N$-hydroxysuccinimide ester (BNHS), N,N-Dimethylformamide (DMF), $\mathrm{N}$ Hydroxysuccinimide (NHS), N,N'-dicyclohexylcarbodiimide (DCC), dimethyl slfoxide (DMSO), hydrogen peroxide, coomassie brilliant blue G250, Tween 20, complete and incomplete Freund/s adjuvant (CFA and IFA), 3,3',5,5'tetramethylbenzidine (TMB) were purchased from Sinopharm, China. Streptavidin-horseradish peroxidase(SA-HRP) was purchased from Sangon Biotech, China. All reagents were of analytical grade unless specified otherwise.

${ }^{1} \mathrm{H}$ Nuclear Magnetic Resonance Spectrometer (NMR) was obtained on a Avance III $400 \mathrm{MHz}$ instrument (Bruker, Switzerland) in $\mathrm{CDCl}_{3}$ solution. Fourier Transform infrared spectrometer (FTIR) was detected by a Nicolet6700 instrument (Thermo, USA). A Multiskan MK3 ELISA reader (Thermo, USA) was applied for the determination of absorbance in dual wavelength mode $(450 / 650 \mathrm{~nm})$, with polystyrene 96-well microplates, purchased from Sangon Biotech (Shanghai, China).TBBPA-protein conjugate was characterized by UV-2012 PC spectrophotometer (UNICO, USA). The ultrapure water used was prepared by Milli-Q system (Millipore, Bedford, MA).

For HPLC analysis, an Agilent LC1100 HPLC system equipped with an Agilent Eclipse Plus C18 (250 mm×4.6 mm, $5 \mu \mathrm{m})$ was used. The mobile phase was Methanol: water $(80: 20, \mathrm{v} / \mathrm{v})$ at a flow rate of $0.3 \mathrm{~mL} / \mathrm{min}$. The injection volume was $20 \mu \mathrm{L}$ and detected at $280 \mathrm{~nm}$.

\subsection{Synthesis of TBBPA hapten}

Tetrabromobisphenol A molecule does not contain functional groups that can connect with proteins directly. So, TBBPA hapten must be synthesized firstly. The synthesized route was illustrated in Fig.1. Synthesis procedures and characterization date of hapten was given below. 


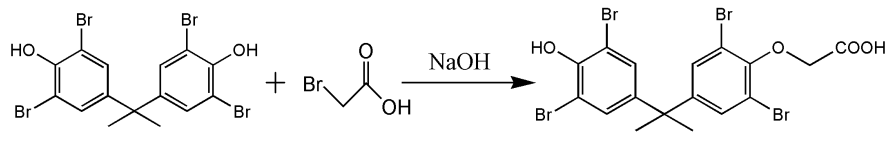

Fig.1 Synthetic route of TBBPA hapten

\subsection{Preparation of immunogen and coating antigen}

The activated ester method and mixed anhydride method were employed for coupling hapten to carrier proteins, using the terminal carboxyl group on TBBPA hapten molecule. The prepared hapten-proteins conjugates, BSA-TBBPA and OVATBBPA, were used as immunogen and coating antigen, respectively.

All the protein conjugates were identified by UV-Vis spectrophotometer and the coupling ratios could be estimated by the mole absorbance $\varepsilon^{[14]}$ and calculated by Eq. 1 .

The coupling ratio $=\frac{\varepsilon_{\text {conjugate }}-\varepsilon_{\text {protein }}}{\varepsilon_{\text {hapten }}} \ldots . .$. Eq. 1

\subsection{Preparation of biotinlyated antibody}

Rabbit polyclonal anti-TBBPA antibodies (pAb-TBBPA) were prepared as described in previously report of out research team ${ }^{[15]}$. After immunized by TBBPA-BSA for four months, the polyclonal antibodies were separated and purification from rabbit serum. Biotinylated TBBPA antibodies (Bi-pAbTBBPA) were prepared as following: $5.0 \mathrm{mg}$ purified $\mathrm{pAb}-$ TBBPA was dissolved to $1.0-2.0 \mathrm{mg} / \mathrm{mL}$ by $0.1 \mathrm{~mol} / \mathrm{L}$ sodium carbonate buffer (CBS, pH 9.6). The antibody solution was mixed with $1.0 \mathrm{mg} / \mathrm{mL}$ BNHS in DMSO at the mass ratio of 1:10. The mixture was stirred for $4 \mathrm{~h}$ and then dialyzed against PBS for $3 \mathrm{~d}$. The as-obtained biotinlated antibody was stored at $4^{\circ} \mathrm{C}$ before use.

\subsection{Heterologous indirect competitive BA-ELISA}

BA-ELISA determinations were performed in 96-well microplate in indirect format. A microplate was coated at $4^{\circ} \mathrm{C}$ overnight with $100 \mu \mathrm{L} /$ well of coating solution. After washing (3 times), unbound active sites were blocked by $200 \mu \mathrm{L} /$ well blocking reagent, later incubation at $37^{\circ} \mathrm{C}$ for $60 \mathrm{~min}$. After a further washing step, standard or sample $(50 \mu \mathrm{L} /$ well $)$ and biotinylated antibody (50 $\mu \mathrm{L} /$ well) were added and incubated for $90 \mathrm{~min}$ at $37^{\circ} \mathrm{C}$.After an additional washing step, $100 \mu \mathrm{L} /$ well SA-HRP conjugate (dilution 1:1000) was added and incubated for $60 \mathrm{~min}$. After a final washing step, $100 \mu \mathrm{L} /$ well of the substrate solution TMB was added, and after sufficient color development (after $15 \mathrm{~min}$ ), the enzymatic reaction was stopped by the addition of $50 \mu \mathrm{L} /$ well of $2 \mathrm{~mol} / \mathrm{L} \mathrm{H}_{2} \mathrm{SO}_{4}$. The absorbance of each well was read in dual-wavelength mode, $450 \mathrm{~nm}$ as test and $650 \mathrm{~nm}$ as reference.

\subsection{Immunoassay optimization}

For developing a sensitive immunoassay method, several parameters were optimized. Firstly, the concentrations of Bi-pAb-TBBPA and coating antigen were determined by checkerboard. In immunoassay method procedures, the optimal blocking solution should exhibit the lowest background value. Different blocking solutions, such as gelatin, OVA, milk power, $\mathrm{PEG}_{20000}$ and PVA, were dissolved in PBS buffer which resulted background values were compared. For discussion the influences of different ionic strength, $\mathrm{pH}$ and matrix effect, competitive curves were performed from TBBPA standards and relative antibody dissolved in PBS buffer. All determinations were performed in triplicate, and the mean absorbance values were processed. Under optimal condition, the calibration curve of BA-ELISA was set up by plotting inhibition against the logarithm of the standard concentration, where, inhibition (\%) was calculated as Eq.2.

$$
\text { Inhibition }(\%)=\frac{\left(A_{\max }-A_{\min }\right)-\left(A_{s}-A_{\min }\right)}{A_{\max }-A_{\min }} \times 100 \%
$$

......Eq.2

Where $A_{\max }$ was absorbance in absence of TBBPA; $A_{\text {min }}$ was the absorbance of black sample; $A_{s}$ was the absorbance of TBBPA at standard concentration.

The $\mathrm{IC}_{50}$, which represented a concentration of a compound at which it inhibits a particular phenomenon by $50 \%$, was employed to evaluate the sensitivity of method. Analogously, the limit of detection (LOD) was evaluated by the $\mathrm{IC}_{10}$.

\subsection{Cross-reaction}

Specificity of the optimized assay was tested by measuring cross-reactivity (CR) using a group of structural analogs. The CR values were calculated according to Eq.3.

$$
C R(\%)=\frac{I C_{50} \text { of } T \mathrm{BBPA}}{I C_{50} \text { of } \text { analogues }} \times 100 \%
$$

......Eq.3

\subsection{Sample preparation}

TBBPA, as a kind of brominated flame retardants, was widely used in electronic products especially in plastic assemblies. So, some electronic waster samples were collected, which was obtained from common electronic castoff, like computer, cell phone, printer, etc. Before analysis, samples must be broken into small size $(5 \mathrm{~mm} \times$ $5 \mathrm{~mm}$ ). A weight of $0.5 \mathrm{~g}$ sample was further ground into powder after frozen by liquid nitrogen. Add the powders into $25 \mathrm{~mL}$ test tube with $20 \mathrm{~mL}$ dichloromethane and ultrasound for 1 hour. Remove the extract liquor and wash the residue with methanol. Under a gentle nitrogen stream, the organic phase was evaporated to nearly dryness and the residue was redissolved with $5 \mathrm{~mL}$ methanol. After filtered, the filtrate was used for immunoassay and HPLC analysis. Prior to immunoassay, the extracts of solid plastic particulars were diluted with PBST at least 20-fold.

\subsection{HPLC analysis}

In order to verify the results detected by BA-ELISA method. TBBPA concentrations of different samples were also detected by HPLC method (Agilent LC1100) consisted of a high pressure pump, a column thermostat (Model 201TP5415), a variable wavelength ultraviolet detector and a vacuum de-air machine. The temperature of $\mathrm{C} 18$ chromatographic column $(250 \mathrm{~mm} \times 4.6 \mathrm{~mm}, 5 \mu \mathrm{m})$ was 
controlled at $35^{\circ} \mathrm{C}$. The mobile phase is methanol-water (80:20 V/V). $20 \mu \mathrm{L}$ sample solution was injected at a flow rate of 0.3 $\mathrm{mL} / \mathrm{min}$. The detection wavelength is $280 \mathrm{~nm}$.

\section{Results and Discussion}

\subsection{The characterization of immunogen, coating antigen and antibody}

For developing a sensitive and specific immunoassay, TBBPA molecule must be derived for coupling with protein. The phenolic group of TBBPA seemed to be the most convenient group, which acidity makes it easy to generate corresponding alkoxide with sodium hydroxide. A common strategy was to convert the phenolic group to ether, terminated with a carboxylic acid. Using this carboxylic acid to couple with protein, BSA-TBBPA and OVA-TBBPA had been successfully synthesized for immunogen and coating antigen, respectively. The hapten-protein conjugates were characterized by UV-visible spectrophotometer, and the results were exhibited in Fig.2. Meanwhile, the coupling ratio was calculated as eq. 1 .

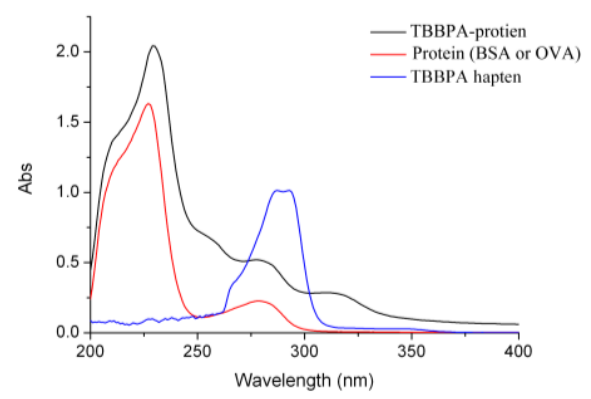

Fig.2 The UV spectra of hapten-protein conjugate

As shown in Fig.2, maximum absorption peaks of TBBPA hapten and protein were appeared at $287 \mathrm{~nm}, 237 \mathrm{~nm}$ and $273 \mathrm{~nm}$, respectively. However the maximum absorption peaks of TBBPA-protein conjugates were appeared at 229, 277 and $317 \mathrm{~nm}$. The displacement of absorption peaks indicated that the hapten was successfully conjugated to the protein. The coupling ratios were 23.1 for BSA-TBBPA, 15.7 for OVA-TBBPA.

The as-obtained BSA-TBBPA artificial antigen was injected into the body of rabbit, and after immunity for 16 weeks; the highest antibody titer is 1:120000. Protein concentrations of immunogen, coating antigen and pAbTBBPA, determined by the coomassie blue method, were $7.69,4.24$ and $17.57 \mathrm{mg} / \mathrm{mL}$, respectively.

\subsection{Suitable operating conditions of immunoassay method}

In order to enhance the sensitivity for immunoassay, the optimum concentration of Bi-pAb-TBBPA and coated antigen is the primary influencing factor. According to checkerboard method, the most optimum reagent concentrations were selected as those that give the maximum absorbance $\left(\mathrm{A}_{0}\right)$ about 1.0 and have the lowest concentration of antibody and coating antigen. The optimal concentration of the coated TTBPA-OVA was $21.2 \mu \mathrm{g} / \mathrm{mL}$, the relative Bi-pAb-TBBPA was in 1:1500 dilutions.
The blocking step is necessary in immunoassay procedures, which helps to eliminate unoccupied sites of the plates. Otherwise, unoccupied sites may absorb the components such as Bi-pAb-TBBPA or SA-HRP during the subsequent steps. Different blocking solutions, gelatin $(0.1 \%$, $0.5 \%), 0.5 \% \mathrm{PEG}_{20000}, 2 \%$ glucan, $1 \% \mathrm{PVA}, 0.5 \%$ OVA or $0.5 \%$ milk power in PBS, were compared, which results were shown in Fig.3a. An optimum blocking reagent should give the lowest background value. Known from the results, $0.5 \%$ gelatin put up the lowest background value (0.09), compared to that of $0.1 \%$ gelatin $(0.13), 0.5 \% \mathrm{PEG}_{20000}(0.17), 0.5 \%$ OVA (0.15), 2\% glucan (0.16), 1\% PVA (0.12), $0.5 \%$ milk pwer (0.16). So, $0.5 \%$ gelatin was selected as blocking agent in the subsequent study.

Heterologous immunosorbent assay is based on the combination of coating conjugate, absorbed on the surface of solid phase, and relative antibody in liquid phase, which combination capacity is widely affected on ionic strength, $\mathrm{pH}$, and other organic matters in liquid phase. Under diverse operating conditions, different $\mathrm{IC}_{50}$, maximal absorbance and $\mathrm{CV}(\%)$ values from competitive curves had been discussed.

The binding reaction of the antibody and antigen is under a dynamic balance and characterized by the weak intermolecular bonds and could be affected by the $\mathrm{pH}$. The $\mathrm{pH}$ value of the optimum assay buffer was adjusted to 5 9. The $\mathrm{IC}_{50}, \mathrm{~A}_{0 \max }$ and $\mathrm{CV}(\%)$ was considered as $\mathrm{pH}$ changing, results were showed in Fig.3c. $\mathrm{A}_{0 \max }$ values were diminished notably when the $\mathrm{pH}$ values were increased. However, the $\mathrm{CV}(\%)$ increased from $5.7 \%(\mathrm{pH} 7.5)$ to $24.33 \%$ (pH 9), meaning that the stability of the immunoassay was reduced when system changed from acidic to alkaline. And the appropriate $\mathrm{IC}_{50}$, $0.59 \mathrm{ng} / \mathrm{mL}$, values were obtained at $\mathrm{pH}$ 7.5. Hence, considering the sensitivity, $\mathrm{pH} 7.5$ is preferred for the buffer of the competition step of the immunoassay.

To evaluate the interference of different matrices on ELISA performance, the matrix effects, caused by common organic solution used in standard solution preparation or sample extraction. The influence results caused by DMSO, methanol an acetone were compared in Fig.3d-f. In DMSO and methanol trial, a clear decrease in sensitivity was observed when the amounts of organic solvents in buffer increasing. The lowest $\mathrm{IC}_{50}$ values were obtained when DMSO and methanol were both at $5 \%(\mathrm{~V} / \mathrm{V}), 0.58 \mathrm{ng} / \mathrm{mL}$ and $0.60 \mathrm{ng} / \mathrm{mL}$, respectively. On the other hand, in acetone trial, the sensitivity and stability of assay were lower than that of other solvent. Known from matrix effects, minimal organic solvent amounts had negative effect on the performance of assay, so sample extracts containing organic solvents need be appropriately diluted in assay buffer to be reliable analyzed.

\subsection{LOD and Working Range}

Under the optimum conditions, the indirect complete ELISA was used for detecting TBBPA, the linear working range, which is determined as the concentrations causing 20$80 \%$ inhibition of color ${ }^{[16]}$, was $0.06-5.89 \mathrm{ng} / \mathrm{mL}$, with linear regression equation $\mathrm{y}=29.8 \lg \mathrm{C}+57.7, \mathrm{R}^{2}=0.968$. The limit of detection (LOD) and sensitivity of the assay for TBBPA, which are represented as $\mathrm{IC}_{10}$ and $\mathrm{IC}_{50}$, were 0.03 and 0.58 $\mathrm{ng} / \mathrm{mL}$, respectively. The working range was analyzed by 
linear regression, plotting the theoretical TBBPA concentrations against measured results by BA-ELISA, with an excellent correlation coefficient $\left(\mathrm{R}^{2}=0.990, \mathrm{n}=8\right)$.

\subsection{Specificity}

Assay specificity indicates the ability of antibody to combine with only target molecule. Cross-reactivity (\%) is an important factor in immunoassay, which is generally used to estimate the specificity of antibody. The cross-reactivity values were calculated according to Eq.3.

3,4-Dichlorobiphenyl $\left(\mathrm{PCB}_{12}\right) 、 3,4,4$ '-Trichlorobiphenyl $\left(\mathrm{PCB}_{37}\right) 、 3,3^{\prime}, 4,4^{\prime}-$ Tetrachlorobiphenyl $\left(\mathrm{PCB}_{77}\right)$ 、 Tris $(2,3-$ dibromopropyl) isocyanurate

(TBC)

Hexabromocyclododecane (HBCD)、Bisphenol A (BPA) were selected as the analogues of TBBPA. The chemical structure of analogues and cross-reactivity results were shown in Tab.1. The results showed that the obtained antibody was specific for TBBPA. And the relative antibody, with highaffinity, was suitable for specific detection TBBPA at low levels.

Tab.1 The cross-reactivity of antibody with ibuprofen and naproxen

\begin{tabular}{ccc}
\hline Analogues & $\mathrm{IC}_{50}(\mathrm{ng} / \mathrm{L})$ & Cross-reactivity $(\%)$ \\
\hline BBPA & $1.396 \times 10^{3}$ & 100 \\
PCB12 & $4.106 \times 10^{4}$ & 0.4 \\
PCB37 & $1.454 \times 10^{3}$ & 1.6 \\
PCB77 & $6.288 \times 10^{3}$ & 12.2 \\
TBC & $>1.396 \times 10^{6}$ & $<0.02$ \\
HBCD & $>6.98 \times 10^{5}$ & $<0.05$ \\
BPA & $9.561 \times 10^{3}$ & 13.6 \\
\hline
\end{tabular}

\subsection{The repeatability of immunoassay method}

Intra-assay repeatability was estimated over 10 replicates and interassay repeatability was evaluated over a months. For estimate the repeatability, the intra-assay coefficient of variation and inter-assay coefficient of variation were calculated. The intra-assay and interassay coefficients of variation $(\mathrm{CV})$ were $<10.51 \%$ and $<13.23 \%$, respectively.

\subsection{Determination of TBBPA in electronic waste samples and recovery tests}

The BA-ELISA method was applied to detect TBBPA in practical samples. The levels of TBBPA in electronic waste showed great variations dependent on the species of plastic. The results were exhibited in Tab.2.These practical samples were also tested on HPLC in order to evaluate precision of BA-ELISA. The BA-ELISA results tended to be slightly higher than those of HPLC method.

The recovery of samples spiked with the target analyte was calculated to assess the analytical performance of immunoassay. All the samples were performed three times in duplicate under optimized procedure to verify the repeatability. The results were shown in Tab.3 and the spiked samples were measured by the BA-ELISA as well as HPLC for comparison. The average recoveries and coefficient of variant values (CV) of BA-ELISA were 93.2-117.3\% and 3.7$9.1 \%$, respectively. Meanwhile, HPLC gave the recoveries of 89.6-105.2\% and CVs of 1.3-7.7\%.

Tab.2 The tested results of TBBPA in electronic waste by BAELISA and HPLC

\begin{tabular}{cccc}
\hline Samples* & Materials & $\begin{array}{c}\text { BA-ELISA } \\
\text { results }(\mathrm{mg} / \mathrm{kg})\end{array}$ & $\begin{array}{c}\text { HPLC results } \\
(\mathrm{mg} / \mathrm{kg})\end{array}$ \\
\hline Keyboard Key & PBT & $1871.8 \pm 8.8$ & $1764.3 \pm 0.3$ \\
Presses & PC & $22.7 \pm 6.3$ & $20.1 \pm 2.9$ \\
Phone's shell & ABS & $276.9 \pm 1.4$ & $273.6 \pm 1.5$ \\
Computer's shell & epoxy & $560.2 \pm 6.5$ & $554.3 \pm 1.1$ \\
Circuits \&circuit & resin & $4650.3 \pm 1.2$ & $4432.5 \pm 0.3$ \\
boards & PVC & $178 \pm 10.7$ & $166.6 \pm 0.7$ \\
Plastic insulators & PP & $3464.1 \pm 3.2$ & $3412.3 \pm 1.6$ \\
Capacitance & PS & & \\
Print's shell &
\end{tabular}

*The Dosage limit of TBBPA in electronic products is $<1 \%(10 \mathrm{~g} / \mathrm{kg})$, according to Norway ROHs.

Tab.3 Recoveries of TBBPA form fish meat samples by BA-ELISA and traditional ELISA

\begin{tabular}{ccccc}
\hline Sample & $\begin{array}{c}\text { Sample } \\
\text { concentration } \\
(\mathrm{mg} / \mathrm{kg})\end{array}$ & $\begin{array}{c}\text { Spiked } \\
\text { level } \\
(\mathrm{mg} / \mathrm{kg})\end{array}$ & $\begin{array}{c}\text { Aecovery\% } \pm \text { CV\% }(\mathrm{n}=3) \\
\text { BA- } \\
\text { ELISA }\end{array}$ & HPLC \\
\hline Phone's & 22.7 & 10 & $96.7 \pm 7.3$ & $92.1 \pm 5.6$ \\
shell & & 15 & $104.9 \pm 9.1$ & $102.7 \pm 7.7$ \\
& & 20 & $117.3 \pm 6.4$ & $105.2 \pm 1.3$ \\
Computer's & 276.9 & 100 & $93.2 \pm 4.2$ & $89.6 \pm 2.8$ \\
shell & & 150 & $113.3 \pm 3.7$ & $110.3 \pm 4.4$ \\
& & 200 & $106.3 \pm 5.3$ & $99.8 \pm 2.5$ \\
\hline
\end{tabular}

\section{Conclusion}

In this study, a kind of tetrabromobisphenol A (TBBPA) hapten and relative hapten-protein complexes, immunogen and coationg antigen, were successfully synthesized. The specific antibody was produced and further established a high sensitivity and efficacious immunoassay menthod BA-ELISA for rapid detection of TBBPA in electronic waste samples. This established method has lower limit of detection and higher sensitivity. The linear range was $0.06-5.89 \mathrm{ng} / \mathrm{mL}$. $\mathrm{IC}_{50}$ was $0.58 \mathrm{ng} / \mathrm{mL}$, with negligible cross-reactivity to structural analogs. A series of electronic waste samples as well as spiked ones were tested by established BA-ELISA and HPLC method. A good correlation of tested results had been demonstrated by different methods. The recovery and coefficient of variation of this immunoassay of TBBPA were acceptable. So, the established BA-ELISA method could be widely used for sensitive and selective detecting TBBPA in environment.

\section{Acknowledgements}

We acknowledge the financial support by the National Natural Science Foundation of China (project No. 21477074).

\section{Reference}

[1] J.B. Fini, A. Riu, L. Debrauwer, et al. Parallel biotransformation of tetrabromobisphenol A in Xenopus laevis and mammals: Xenopus 
as a model for endocrine perturbation studies[J]. Toxicol Sci 2012, 125(2):359-367.

[2] E.C. Kibakaya, K. Stephen, M.M. Whalen. Tetrabromobisphenol A has immunosuppressive effects on human natural killer cells[J]. Immunotoxicol. 2009, 6(4): 285-292.

[3] H. Viberg, P. Eriksson. Differences in neonatal neurotoxicity of brominated flame retardants, PBDE 99 and TBBPA, in mice[J]. Toxicology 2011, 289(1): 59-65.

[4] A. Covaci, S. Voorspoels, M.A. Abdallah, et al. Analytical and environmental aspects of the flame retardant tetrabromobisphenol-A and its derivatives[J]. Journal of Chromatography A, 2009, 1216(4): 346-363.

[5] Z. Xie, R. Ebinghaus, R. Lohmann et al. Trace determination of the flame retardant tetrabromobisphenol $\mathrm{A}$ in the atmosphere by gas chromatography-mass spectrometry[J]. 2007, 584(1):333-342.

[6] X.L. Zhang, X.J. Luo, S.J. Chen, et al. Spatial distribution and vertical profile of polybrominated diphenyl ethers, tetrabromobisphenol $\mathrm{A}$, and decabromodiphenylethane in river sediment from an industrialized region of South China[J]. Environmental Pollution 2009, 157(6): 1917-1923.

[7] Z.X. Shi, Y.N. Wu, J.G. Li, Y.F. Zhao, J.F. Feng. Dietary exposure assessment of Chinese adults and nursing infants to tetrabromobisphenol-A and hexabromocyclododecanes: Occurrence measurements in foods and human milk[J]. Environ. Sci. Technol. 2009, 43(12): 4314-4319.

[8] P. Guerra, E. Eliarrat, D. Barceló. Simultaneous determination of hexabromocyclododecan, tetrabromoisphenol $\mathrm{A}$, and related compounds in sewage sludge and sediment samples from Ebro River basin (Spain)[J]. Anal. Bioanal. Chem. 2010, 397(7): 2817-2824.

[9] W. L. Shelver, Y.S. Keum, H.J. Kim, D. Rutherford, et al. Hapten Syntheses and Antibody Generation for the Development of a Polybrominated Flame Retardant ELISA $\|[\mathrm{J}]$. Journal of agricultural and food chemistry[J]. Agric. Food Chem. 2005, 53(10): 3840-3847

[10] W.L. Shelver, C.D. Parrotta, R. Slawecki, Q.X. Li, et al. Development of a magnetic particle immunoassay for polybrominated diphenyl ether and application to environmental and food matrices[J]. Chemosphere 2008, 73(1): S18-S23.

[11] K.C. Ahn, S.J. Gee, H.J. Tsai, D. Bennett, et al. Immunoassay for Monitoring Environmental and Human Exposure to the Polybrominated Diphenyl Ether BDE-47[J].Environ. Sci. Technol. 2009, 43(20): 7784-7790.

[12] H.J. Kim, M.A. Rossotti, K.C. Ahn, et al. Development of a noncompetitive phage anti-immunocomplex assay for brominated diphenyl ether 47[J]. Anal. Biochem. 2010, 401(1): 38-46.

[13] J. Wang, H. Li, W.L. Shelver, et al. Development of a monoclonal antibody-based, congener-specific and solvent-tolerable direct enzyme-linked immunosorbent assay for the detection of 2,2 ', 4,4 '-tetrabromodiphenyl ether in environmental samples[J]. Anal. Bioanal. Chem. 2011, 401(7): 2249-2258.

[14] N. Liu, P. Su, Z.X. Gao, M.X. Zhu, et al. Simultaneous detection for three kinds of veterinary drugs: chloramphenicol, clenbuterol and 17-beta-estradiol by high-throughput suspension array technology[J]. Anal. Chim. Acta 2009, 632(1): 128-134.

[15] H.Y. Chen, H.S. Zhuang, G.X. Yang, Development of a new polyclonal antibody for the determination of polychlorinated biphenyls in indoor air by ic-ELISA[J]. Environmental science and pollution research, 2013, 20(4):2244-2251.

[16] S. Wang, R.D. Allan, J.H. Skerritt, I.R. Kennedy. Development of a class-specific competitive ELISA for the benzoylphenylurea insecticides[J]. Agric. Food Chem. 1998, 46(8): 3330-3338. 